\title{
Mechanics of chondrocyte hypertrophy
}

\author{
C. C. van Donkelaar • W. Wilson
}

Received: 23 March 2011 / Accepted: 29 July 2011 / Published online: 17 August 2011

(C) The Author(s) 2011. This article is published with open access at Springerlink.com

\begin{abstract}
Chondrocyte hypertrophy is a characteristic of osteoarthritis and dominates bone growth. Intra- and extracellular changes that are known to be induced by metabolically active hypertrophic chondrocytes are known to contribute to hypertrophy. However, it is unknown to which extent these mechanical conditions together can be held responsible for the total magnitude of hypertrophy. The present paper aims to provide a quantitative, mechanically sound answer to that question. To address this aim requires a quantitative tool that captures the mechanical effects of collagen and proteoglycans, allows temporal changes in tissue composition, and can compute cell and tissue deformations. These requirements are met in our numerical model that is validated for articular cartilage mechanics, which we apply to quantitatively explain a range of experimental observations related to hypertrophy. After validating the numerical approach for studying hypertrophy, the model is applied to evaluate the direct mechanical effects of axial tension and compression on hypertrophy (Hueter-Volkmann principle) and to explore why hypertrophy is reduced in case of partially or fully compromised proteoglycan expression. Finally, a mechanical explanation is provided for the observation that chondrocytes do not hypertrophy when enzymatical collagen degradation is prohibited (S1Pcko knock-out mouse model). This paper shows that matrix turnover by metabolically active chondrocytes, together with externally applied mechanical
\end{abstract}

Electronic supplementary material The online version of this article (doi:10.1007/s10237-011-0340-0) contains supplementary material, which is available to authorized users.

C. C. van Donkelaar $(\varangle) \cdot$ W. Wilson

Department of Biomedical Engineering,

Eindhoven University of Technology, PO Box 513,

5600 MB Eindhoven, The Netherlands

e-mail: c.c.v.donkelaar@tue.nl

URL: http//www.mate.tue.nl/mate/showemp.php/65 conditions, can explain quantitatively the volumetric change of chondrocytes during hypertrophy. It provides a mechanistic explanation for the observation that collagen degradation results in chondrocyte hypertrophy, both under physiological and pathological conditions.

Keywords Chondrocyte hypertrophy - Collagen turnover · Tissue remodeling · Osmotic swelling •

Finite element model

\section{Introduction}

Chondrocyte hypertrophy is one of the characteristics of osteoarthritis (OA) (Kirsch et al. 2000; Tchetina et al. 2007; van der Kraan et al. 2009), and therefore a potential therapeutic target. Also, various growth pathologies are associated with abnormal hypertrophy (Kuizon and Salusky 2004; Wilkie 2005; Zuscik et al. 2002). During hypertrophy, collagenase activity and cellular and matrix changes are known to occur concurrently (Ortega et al. 2004; Tchetina et al. 2007). The intracellular volumes occupied by organelles and cytoplasmic ground substance gradually increase (Buckwalter et al. 1986), together with the osmotic pressure generated by intracellular accumulation of organic osmolites (Farnum et al. 2002). Although quantification of the effects of cellular electro-chemo-mechanical properties has proven difficult, it is apparent that this can only be partially responsible for the tenfold volume change and four to five times increased cell height during hypertrophy (Buckwalter et al. 1986; Hunziker and Schenk 1989; Noonan et al. 1998; Wilsman et al. 1996). Interestingly, to keep up with such increase in cell height, the aligning extracellular matrix (ECM) needs to also stretch to $400-500 \%$ its original length. Tissues would fail if they were passively strained to such magnitudes. The mechanisms 


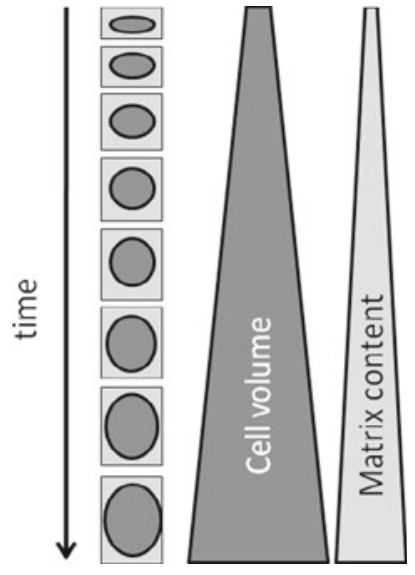

Fig. 1 Left Schematic of the change in cell shape and volume (dark circle) within the tissue over time. Middle Cell volume increases multiplefold in time. Right the matrix content (both proteoglycan and collagen) that is associated with one cell increases over time. The change in matrix volume is, however, smaller than the change in cell volume. Hence, the ratio of cell volume over matrix volume increases during hypertrophy

behind the impressive increases in cell volume and tissue elongation are yet unexplained. The biomechanical nature of these phenomena warrants a thorough, quantitative evaluation of mechanical principles underlying this mechanism.

Changes to the extracellular and intracellular constitution are key to understand the biomechanics of hypertrophy. The combined effect of changes in cell volume and matrix constitution is that the volume occupied by cells over the volume of ECM decreases from the early proliferative to the late hypertrophic zone. Yet, the total amount of matrix associated with one hypertrophic cell increases, with values ranging from 50 to $1000 \%$ depending on species, age and growth plate (Buckwalter et al. 1986; Farnum et al. 2002; Hunziker and Schenk 1989; Wilsman et al. 1996) (Fig. 1). The fraction of large proteoglycans per volume of matrix is largest around hypertrophic cells (Maroudas et al. 1991), which is especially interesting given that hyaluronan-mediated hydrostatic pressure causes lacuna expansion (Gakunga et al. 2000). Active proteoglycan metabolism in this area is illustrated by an up-regulation of hyaluronidases, ADAMTS-5 (Sztrolovics et al. 2002) and ADAMTS-9 (Kumagishi et al. 2008).

Hypertrophic cells show active collagen metabolism. MMP's are abundant in quantity and diversity around hypertrophic cells, while these cells continue synthesizing collagen [review by (Ortega et al. 2004)]. Consequently, the fraction of fibrillar collagen per volume of matrix from the upper proliferative to the lower hypertrophic zones in both the pericellular and interterritorial matrices increase in 4-5-month-old miniature pigs (Noonan et al. 1998), but decrease in newborn mice (Buckwalter et al. 1986). Collagen degradation in the hypertrophic zone decreases collagen-II fraction in bovine fetuses, associated with a loss of collagen network integrity, while collagen-X content increases (Alini et al. 1992). This has lead to the hypothesis that collagen turnover is an ultimate prerequisite to enable hypertrophy. This thought is reinforced by the finding that chondrocytes do not hypertrophy in S1Pcko knock-out mice, which are unable to enzymatically degrade collagen-IIB, even though markers for hypertrophy such as collagen- $X$ are normally expressed and the Ihh-PTHrP pathway is unaffected (Patra et al. 2006a,b).

All of the above mentioned intracellular and extracellular conditions are thought to contribute to chondrocyte hypertrophy. However, it is unknown whether these conditions together can induce the amount of hypertrophy that occurs in chondrocytes. The aim of the present study is to provide a quantitative, mechanically sound answer to that question. Also, it aims to provide a mechanistic explanation for the relationship between hypertrophy and collagen turnover.

This requires a quantitative tool that captures the mechanical behavior of collagen and proteoglycans, allows temporal changes in tissue composition, and can compute cell and tissue deformations. Therefore, we adapt our model for cartilage mechanics (Wilson et al. 2006b, 2007, 2005, 2004), such that proteoglycan and collagen concentrations can change with time, and we implement a description for collagen turnover. Input parameters are obtained from epiphyseal plate studies, and unknown parameters are fitted to experimental data. After corroborating model predictions with the literature, we alter selective parameters and compare predictions with effects that are known to occur experimentally. These include extended simulations to accommodate more extreme terminal cell sizes (Hunziker 1994), application of axial tension or compression to corroborate the Hueter-Volkmann principle, which says that bone growth in epiphyseal plates is load-dependently reduced by compression and enhanced by tension and is predominantly the results of changes in the rate of hypertrophy and the ultimate size of hypertrophic cells (Stokes 2002; Stokes et al. 2007, 2005), and simulations of hypertrophy in case of partially or fully compromised proteoglycan expression (Vanky et al. 2000). Finally, we explore how mechanical conditions may be involved in the aforementioned S1Pcko knock-out mouse model in which cells do not hypertrophy (Patra et al. 2006a,b).

\section{Methods}

\subsection{Model description}

We apply our model, originally developed to capture the mechanical behavior of articular cartilage, to a single hypertrophying chondrocyte and its associated matrix. The computational tool uses the finite element method and includes mechanical effects of swelling pressure by charged proteoglycans, as well as constraints against swelling by a 
Table 1 Properties used for cell, pericellular matrix (PCM), interterritorial matrix (ITM) and collagen fibers

\begin{tabular}{|c|c|c|c|c|c|}
\hline & Cell & PCM & ITM & Fibers & Equations \\
\hline \multicolumn{6}{|c|}{ Mechanical parameters } \\
\hline$E_{m}(\mathrm{MPa})$ & $0.018^{1}$ & $0.0903^{2,3}$ & $0.903^{4}$ & & $(4,5)$ \\
\hline$E_{f}(\mathrm{MPa})$ & & & & $4.316^{5}$ & (2) \\
\hline$k_{f}(-)$ & & & & $16.85^{5}$ & (2) \\
\hline$c_{\mathrm{PG}}(\mathrm{meq} / \mathrm{ml})$ & $0.467^{\mathrm{b}}$ & $0.61^{6}$ & $0.61^{6}$ & & (9) \\
\hline \multicolumn{6}{|c|}{ Physiological parameters } \\
\hline$d V_{\text {coll }}\left(\mu \mathrm{m}^{3}\right)^{\mathrm{a}}$ & & $0.079^{7}$ & $0.059^{7}$ & & (17) \\
\hline$d V_{\mathrm{PG}}\left(\mu \mathrm{m}^{3}\right)^{\mathrm{a}}$ & $5.88 \mathrm{e}-3^{\mathrm{b}}$ & $0.21^{7}$ & $0.065^{7}$ & & (18) \\
\hline$n_{\mathrm{PG}, 0}(-)$ & $0.3^{\mathrm{b}}$ & $0.172^{7}$ & $0.154^{7}$ & & (19) \\
\hline$n_{\text {coll }, 0}(-)$ & & $0.131^{7}$ & $0.148^{7}$ & & (19) \\
\hline
\end{tabular}

a Added volumes during the terminal differentiation

${ }^{\mathrm{b}}$ Quantities could not be derived from the literature and are fitted

${ }^{1}$ Guilak et al. (2002), ${ }^{2}$ Alexopoulos et al. (2003), ${ }^{3}$ Guilak et al. (2005),

${ }^{4}$ Radhakrishnan et al. (2004), ${ }^{5}$ Wilson et al. (2006b), ${ }^{6}$ Narmoneva et al. (1999), ${ }^{7}$ Noonan et al. (1998)

reinforcing network of collagen fibrils. The total tissue stress $\left(\sigma_{\text {tot }}\right)$ is given by (Wilson et al. 2006b, 2007, 2005, 2004)

$$
\begin{aligned}
\sigma_{\mathrm{tot}}= & -\left(\mu^{f} \Delta \pi\right) \boldsymbol{I} \\
& +n_{\mathrm{s}, 0}\left(\left(1-\sum_{i=1}^{\text {totf }} \rho_{\mathrm{c}}^{i}\right) \sigma_{n f}+\sum_{i=1}^{\text {totf }} \rho_{\mathrm{c}}^{i} \sigma_{\mathrm{f}}^{i} \vec{e}_{\mathrm{f}}^{i} \vec{e}_{\mathrm{f}}^{i}\right)
\end{aligned}
$$

where $\mu^{\mathrm{f}}$ is the water chemical potential, $\Delta \pi$ the osmotic pressure gradient, $\boldsymbol{I}$ the unit tensor, $n_{\mathrm{s}, 0}$ the initial solid volume (in the unloaded and non-swollen state), totf the total number of fibers included, $\sigma_{n f}$ the stress in the non-fibrillar matrix, $\sigma_{\mathrm{f}}^{i}$ the fibril stress, $\vec{e}_{\mathrm{f}}^{i}$ the fibril direction, $\rho_{\mathrm{c}}^{i}$ the volume fraction of the fibrils with respect to the total solid volume, and $i$ the number of the fiber compartment. The fibrils are assumed to behave non-linear elastic in tension in the fiber direction only, according to

$\sigma_{\mathrm{f}}^{i}=E_{\mathrm{f}}\left(e^{k_{\mathrm{f}} \varepsilon_{\mathrm{f}}^{i}}-1\right)$

where $E_{\mathrm{f}}$ and $k_{\mathrm{f}}$ are constants describing the material behavior and $\varepsilon_{\mathrm{f}}^{i}$ the strain in the fiber direction. These constants, as well as those in following equations (Table 1) have been thoroughly validated in the past (Wilson et al. 2006b) and were adopted by other groups (Saarakkala et al. 2010).

For the non-fibrillar matrix stiffness we use a compressible neo-Hookean model, in which

$\sigma_{n \mathrm{f}}=K_{\mathrm{m}} \frac{\ln (J)}{J} \boldsymbol{I}+\frac{G_{\mathrm{m}}}{J}\left(\boldsymbol{F} \cdot \boldsymbol{F}^{T}-J^{\frac{2}{3}} \boldsymbol{I}\right)$, where $J$ is the determinant of the deformation tensor $\boldsymbol{F}$, and bulk $(\mathrm{Km})$ and shear moduli $(\mathrm{Gm})$ are defined as

$\begin{aligned} K_{\mathrm{m}} & =\frac{E_{\mathrm{m}}}{3\left(1-2 v_{\mathrm{m}}\right)} \\ G_{\mathrm{m}} & =\frac{E_{\mathrm{m}}}{2\left(1+v_{\mathrm{m}}\right)},\end{aligned}$

where $E_{\mathrm{m}}$ is the Young's modulus and $v_{\mathrm{m}}$ the poisson's ratio.

We assume that the osmotic swelling pressure $(\Delta \pi)$ depends on the number of fixed charges in the proteoglycans per amount of extrafibrillar water (Wilson et al. 2007), as given by

$$
\begin{aligned}
\Delta \pi= & \Phi_{\mathrm{int}} R T\left(\sqrt{c_{\mathrm{F}, \mathrm{exf}}^{2}+4 \frac{\left(\gamma_{\mathrm{ext}}^{ \pm}\right)^{2}}{\left(\gamma_{\mathrm{int}}^{ \pm}\right)^{2}} c_{\mathrm{ext}}^{2}}\right) \\
& -2 \Phi_{\mathrm{ext}} R T c_{\mathrm{ext}}
\end{aligned}
$$

where $\Phi_{\alpha}$ and $\gamma_{\mathrm{a}}$ represent the osmotic and activity coefficients, respectively, which were implemented before (Wilson et al. 2006b) as proposed by Huyghe et al. (2003). $R$ is the gas constant, $T$ the absolute temperature, $c_{\text {ext }}$ the external salt concentration and $c_{\mathrm{F}, \text { exf }}$ the fixed charge density related to the extrafibrillar fluid compartment, which is determined as

$c_{\mathrm{F}, \mathrm{exf}}=\frac{c_{\mathrm{F}} n_{\mathrm{f}}}{n_{\mathrm{exf}}}$

The extrafibrillar fluid fraction ( $n_{\text {exf }}$ is defined based on previous experimental work (Maroudas and Bannon 1981; Maroudas et al. 1991; Urban and McMullin 1988). The implementation was explained in our earlier work (Wilson et al. 2006b, 2007).

The total fixed charge density $\left(c_{\mathrm{F}}\right)$ is assumed to relate linearly to the proteoglycan content in the tissue $\left(n_{\mathrm{PG}}\right)$ via constant $c_{\mathrm{PG}}$, using given known valencies and molecular weights of chondroitin and keratin sulfate chains, and calibrated for the relative ratio at which these two proteoglycan types would normally occur (Narmoneva et al. 1999). In addition, as a consequence of mechanical loading or tissue expansion, the amount of fixed charges per water volume may change. To account for these effects, the fixed charge density is defined as:

$c_{\mathrm{F}}=\frac{c_{\mathrm{PG}} n_{\mathrm{PG}}}{n_{\mathrm{f}}}$

The same equation is used to determine intracellular pressure, by determining intracellular charges $\left(c_{\mathrm{F}}\right)$ in negatively charged intracellular proteins $\left(n_{\mathrm{PG}}\right)$ (Guilak et al. 2002) using a different value for constant $c_{\mathrm{PG}}$.

Because swelling properties are included, an initial equilibration step is necessary during which the tissue swells until swelling pressure reaches a mechanical equilibrium with the tension in the strained non-fibrillar and fibrillar matrix. This step starts at $t=0$, referred to as the initial condition. The 
end of this step is referred to as the equilibrium condition, resembling the late proliferative zone. After equilibrium is reached, hypertrophy and matrix synthesis are started.

\subsection{Matrix synthesis}

Extracellular amounts of collagen and non-fibrillar matrix have been measured at different time points during chondrocyte differentiation in epiphyseal plates. These are converted into values for synthesis per day by linear interpolation. In homogenization approaches such as the one used here, volume-fractions are used rather than actual volumes. The sum of all fractions adds up to one. Hence, assuming all solid matrix consists of collagen and non-fibrilar matrix, and assuming the non-fibrillar matrix consists of proteoglycans,

$n_{\mathrm{f}}+n_{\mathrm{S}}=n_{\mathrm{f}}+n_{\mathrm{coll}}+n_{\mathrm{PG}}=1$

with $n$ the fraction of the constituent and subscripts $\mathrm{f}, \mathrm{s}$, coll and PG representing fluid, solid, collagen and proteoglycans. Depending on the source of the tissue, the matrix may contain an amount of other proteins, which also have been shown to contribute to the mechanical behavior of cartilage (Williamson et al. 2001). We have not included this residual fraction separately when we fitted material parameters in the past (Wilson et al. 2007). Any contribution of this fraction is accounted for in the description of the non-fibrillar matrix. Hence, we assume that the total volume of the solid part of the matrix $\left(V_{\mathrm{s}}\right)$ is occupied by collagen and proteoglycans,

$V_{\mathrm{s}}=V_{\mathrm{coll}}+V_{\mathrm{PG}}$

and the remaining volume is fluid

$V_{\mathrm{f}}=1-V_{\mathrm{s}}$

Fractions of constituents can then be related to their respective volumes as:

$$
\begin{aligned}
& n_{\mathrm{f}}=V_{\mathrm{f}} /\left(V_{\mathrm{f}}+V_{\mathrm{s}}\right) \\
& n_{\mathrm{coll}}=V_{\text {coll }} /\left(V_{\mathrm{f}}+V_{\mathrm{s}}\right) \\
& n_{\mathrm{PG}}=V_{\mathrm{PG}} /\left(V_{\mathrm{f}}+V_{\mathrm{s}}\right) \\
& n_{\mathrm{s}}=V_{\mathrm{s}} /\left(V_{\mathrm{f}}+V_{\mathrm{s}}\right)
\end{aligned}
$$

In this way it is possible to derive volume-fractions from known volumes, but not vice versa. To enable using volumes as well as volume-fractions requires the possibility to go both ways. To accomplish that, a reference equilibrium volume $V_{\text {eq }}$ for each element is to be defined. This is arbitrarily chosen to equal the volume $(J)$ in the mechanical equilibrium condition prior to hypertrophy.

$$
V_{\mathrm{eq}}=J\left(i_{\mathrm{eq}}\right)
$$

where $i_{\text {eq }}$ represents time-step $i$ at which equilibrium is reached, but hypertrophy is not started. Throughout the simulation, we keep track of the volumes of proteoglycans and collagen.

Matrix synthesis is then prescribed by adding a volume of collagen $\left(\mathrm{d} V_{\text {coll }}\right)$ and/or proteoglycans $\left(\mathrm{d} V_{\mathrm{PG}}\right)$ at each timestep:

$$
\begin{aligned}
V_{\text {coll }, i} & =V_{\text {coll }, i-1}+\mathrm{d} V_{\text {coll }} \\
V_{\mathrm{PG}, i} & =V_{\mathrm{PG}, i-1}+\mathrm{d} V_{\mathrm{PG}}
\end{aligned}
$$

To compute the effect of these changes on the structure, the initial solid and fluid fractions as well as the initial fixed charge density are updated using the current $(t=i)$ and initial $(t=0)$ solid volumes and the equilibrium volume:

$n_{\mathrm{s}, 0}=\left(V_{\mathrm{PG}, i}+V_{\mathrm{coll}, i}\right)^{*} V_{\mathrm{eq}}$

$n_{\mathrm{f}, 0}=1-n_{\mathrm{s}, 0}$

$c_{\mathrm{F}, 0}=c_{\mathrm{F}}{ }^{*}\left(n_{\mathrm{f}, 0}-1+J\right) / n_{\mathrm{f}, 0}$

By adding proteoglycans and/or collagen in this way, the newly derived initial solid fraction has become higher on the expense of the fluid fraction, while tissue volume does not change. During subsequent equilibration, total tissue volume increases as the fluid fraction is being restored. The increased total volume in this new equilibrium represents tissue growth due to matrix synthesis during this time-step.

\subsection{Cell hypertrophy}

To accommodate for changes in charged intracellular protein content during hypertrophy, the same equations as for matrix swelling are used, with the subscript PG representing intracellular proteins rather than extracellular proteoglycans.

\subsection{Collagen turnover}

Collagen turnover was rationalized into a continuum description as follows. Catalytic enzymes are assumed to cleave a particular fraction of the collagen during a given time period. Meanwhile, collagen synthesis is ongoing. It is then assumed that newly formed collagen either self-assembles in an unstrained state, or otherwise induces changes in collagen fibrils, such that their strain is ameliorated.

To implement this in the model, the total collagen content is subdivided into a discrete number of compartments. These are not physically meaningful; each compartment is a continuum, they exist at the same location at the same time, and together they represent all the collagen at that location. Yet, the content of each compartment can independently turn over. For example, if ten equal compartments were defined, a change to one compartment represents a change to $10 \%$ of the total collagen mass. If the total collagen content were to increase $0.5 \%$, then this could be obtained by an update of $0.5 \%$ in each compartment, or $5 \%$ in one of them. The rationale to have multiple compartments is that each of them 
could contain fibers of different age, i.e., fibers that exhibit different levels of strain in a growing tissue. Initially, the total collagen volume $\left(V_{\text {coll }}\right)$ is equally subdivided over all defined compartments as

$V_{\text {ncoll }}=V_{\text {coll }} / N$

in which $N$ equals the total number of collagen compartments and $V_{\text {coll }}$ is the volume of compartment ncoll. One compartment is assumed to change per time-step. Hence, changes in collagen volume ( $\mathrm{d} V_{\text {coll }}$ in Eq. 17$)$ in a particular time-step are ascribed to one single compartment, changing Eq. 17 into

$V_{\text {ncoll }, i}=V_{\text {ncoll }, i-1}+\mathrm{d} V_{\text {coll }}$

and the total collagen content in the solid equals

$V_{\text {coll }}=\sum_{\text {ncoll }=1}^{N} V_{\text {ncoll }}$

Each time-step, changes are applied to a different compartment.

To accommodate for the assumption that collagen assembles under unstrained conditions, the length of the fibers in the subjected collagen compartment $L_{\mathrm{ncoll}, 0}$ are updated to the length $L_{\text {ncoll }}$ at the time $i$ at which they are synthesized:

$L_{\mathrm{ncoll}, 0}=L_{\mathrm{ncoll}, i}$.

\subsection{Input parameters}

The parameter values describing the mechanical behavior of the cell and its environment, and the biological parameters characterizing the synthesis of proteoglycans and collagen in the pericellular (PCM) and the interterritorial matrix (ITM) are obtained from the literature (Table 1). The effective hydrophilic property of intracellular proteins and the change thereof with ongoing cell differentiation are estimated. The time-scales relevant to matrix synthesis and hypertrophy are days, while mechanical visco-elastic effects and fluid transport occur over minutes. Also, the coupling between matrix synthesis and hypertrophy is through mechanical effects: enhanced solid content (proteoglycans and collagen) and increased swelling pressure by proteoglycans. To capture the biological processes, we therefore used time-steps equivalent to 1 day periods, and visco-elastic effects are neglected. Hence, we used non-linear elastic descriptions for both the fibrillar and the non-fibrillar solid parts, and we kept hydrostatic pressure at $0 \mathrm{~Pa}$, which means that the distribution and flow of fluid are governed by the osmotic pressure but not by hydrostatic pressure.

The constants $E_{\mathrm{f}}$ and $k_{\mathrm{f}}$ characterizing collagen stiffness are similar to values previously determined for collagen-II in articular cartilage (Wilson et al. 2006b). Stiffness of the nonfibrillar solid is chosen $0.90 \mathrm{MPa}$ for the ITM, based on measurements of matrix stiffness in the resting zone, early and late hypertrophic zones of $0.57,0.88$ and $1.15 \mathrm{MPa}$, respectively (Radhakrishnan et al. 2004). The PCM for articular chondrocytes was determined 24 (Guilak et al. 2005) and $69 \mathrm{kPa}$ (Alexopoulos et al. 2003), roughly an order of magnitude weaker than ITM. We therefore assumed PCM stiffness around the early hypertrophic cell to be 10 times less than the ITM stiffness.

The proteoglycan-rich non-fibrillar solid matrix is assumed to be amorphous. The constant $c_{\mathrm{PG}}$ relating proteoglycan content to fixed charge density is 0.61 (Narmoneva et al. 1999). In the PCM, collagen does not show a predominant orientation, while in the ITM the collagen is strongly aligned with the direction of tissue growth (e.g., Hunziker 1994). Accordingly, we assume an isotropic collagen structure in the PCM and an organized collagen architecture in the ITM, with fibrils aligned with the direction of growth.

Isolated chondrocytes in hypotonic solutions are weaker than chondrocytes under physiological osmotic conditions (Guilak et al. 2002). However, whether these properties apply to in vivo conditions are unknown and reported values for the stiffness of chondrocytes and other cells vary largely. Yet, it is well accepted that cell stiffness is below PCM stiffness. We used 20\% PCM stiffness for the cell. The swelling constant $c_{\mathrm{PG}}$ is unknown for chondrocytes and therefore estimated. The initial water content of a chondrocyte is assumed $70 \%$. Hence, initial $n_{\mathrm{PG}}$ in the cell equals 0.3 .

The base for the finite element simulations is a publication by Noonan et al. (1998), which provides a complete dataset describing chondrocyte differentiation. It contains electron microscopy images of initial and late hypertrophic cell shapes and quantitative measures for the volumes of the cells and their associated PCM and ITM, as well as the collagen and proteoglycan fractions in both PCM and ITM.

Based on these data, an axisymmetric finite element mesh representing the initial cell (274 elements), its amorphous PCM (132 elements) and its fibrous ITM (94 elements) is derived (Fig. 2). Boundary conditions are chosen such that they represent the conditions in Noonan et al. (1998). Assuming that the tissue and cells in the immediate environment behave identically, nodes in the lower plane of the mesh are fixed in vertical direction, nodes along the vertical edges are allowed to move in vertical direction only, and nodes in the top plane are forced to remain horizontally aligned (Fig. 2b).

From the collagen and proteoglycan fractions in the early and late hypertrophic zones (Noonan et al. 1998), the parameters $\mathrm{d} V_{\mathrm{PG}}$ and $\mathrm{d} V_{\text {coll }}($ Eqs. 17, 18) are determined for both PCM and ITM:

$\mathrm{d} V_{X, M}=\frac{V_{X, M}^{\mathrm{LHZ}}-V_{X, M}^{\mathrm{EHZ}}}{T}$, with $X \in\{\mathrm{PG}$, coll $\}$

in which $V^{\mathrm{LHZ}}$ and $V^{\mathrm{EHZ}}$ represent the matrix volumes in the late and early hypertrophic zones, respectively, and $T$ equals 

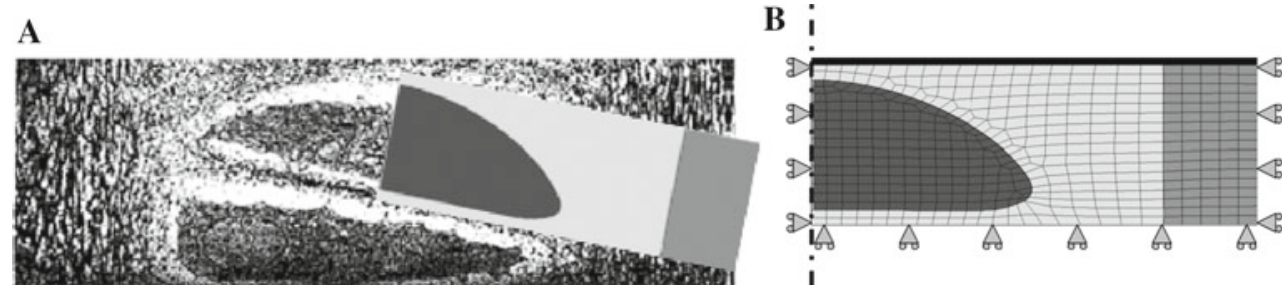

Fig. 2 a Electron microscopy image of a cell from the transition area between the proliferative and early hypertrophic zone of an epiphyseal plate (modified from Noonan et al. 1998), overlaid with the geometry of the initial cell (black), its PCM (light gray) and ITM (dark gray).

the number of time-steps in the simulation. Hence, all input parameters associated with the ECM are known. Parameters $\mathrm{d} V_{\mathrm{PG}}$ and $C_{\mathrm{PG}}$ for the cell, the only unknown input parameters for this simulation, are determined as follows. Initially, we choose the same values that we use for proteoglycans. Then we determine cell size at the initial equilibrium, when $\mathrm{d} V_{\mathrm{PG}}$ equals 0 by definition. At this stage we update $C_{\mathrm{PG}}$ to reach the appropriate cell size. Subsequently, we continue the simulation to the end of hypertrophy and adjusted $\mathrm{d} V_{\mathrm{PG}}$ such that cell size at the end of the simulation also matched the experimental data. Then we linearly interpolated $\mathrm{d} V_{\mathrm{PG}}$ over all increments. These values are kept constant in all subsequent simulations.

\subsection{Simulations}

Cell hypertrophy is simulated by continuously running the model between Eqs. 17 and 25, i.e., by repeatedly adding proteoglycans and collagen, followed by equilibration. Geometric and volumetric data of cell, PCM and ITM are monitored and qualitatively or quantitatively compared with literature data.

First, simulations are performed as described above, without additional boundary conditions. Subsequently, specific conditions are simulated. First, to evaluate the performance of the model with regard to the Hueter-Volkmann principle, additional tensions of $0.1,0.05,-0.05$ and $-0.1 \mathrm{MPa}$ are applied during hypertrophy. Second, the effect of proteoglycan expression is evaluated by simulating hypertrophy when proteoglycans expression fully compromised or reduced to half the normal amount. Third, the effect of MMP's on collagen adaptation is evaluated by prohibiting collagen turnover. All output is compared to relevant literature, further details are provided in the results.

\section{Results}

With the given input parameters, and after iteratively updating the two unknown parameters that describe cell swelling b The finite element mesh with the applied boundary conditions. The ITM contains aligned fibers in the vertical direction, the PCM contains collagen, but without preferred direction

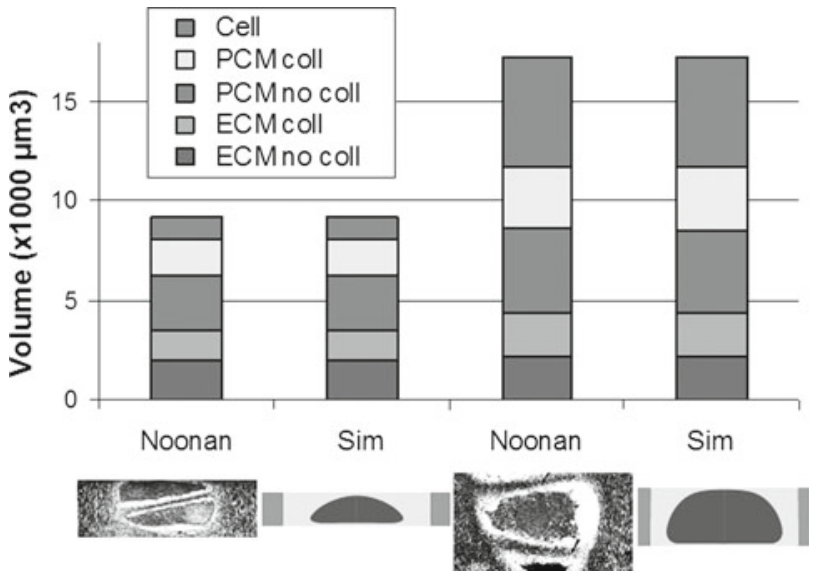

Fig. 3 Comparison between data by Noonan et al. (1998) and simulations, for volumes of the cell and the PCM and ITM, separated out into volume occupied by collagen fibers ('coll') and ground substance ('no coll'). The initial stage (left column) was prescribed into the model (second column). Predicted volumetric changes (right column) concur with the measured components in the late hypertrophic cell (third column). The associated electron microscopy images and the computed geometries are shown below each column

(d $V_{\mathrm{PG}}$ and $C_{\mathrm{PG}}$ (Table 1)), the simulation matched qualitatively and quantitatively the hypertrophic process described by Noonan et al. (1998), including volumes of cell, ITM and PCM, and the collagenous and non-collagenous fractions thereof (Fig. 3). This demonstrates that hypertrophy can indeed result from mechanical changes in the matrix and the cell.

To gain further confidence in the accuracy of model predictions, additional simulations are performed. First, the simulation is extended until the cell reaches 5 times its original height, the size determined for terminal hypertrophic 35-dayold rat proximal tibia chondrocytes. The fivefold increase in cell height (from 7.6 to $38 \mu \mathrm{m}$ ) is accompanied by a total ECM volume increase of 2.7 times (from 2,850 to 7,700 $\mu^{3}$ per cell) (Hunziker 1994). The proliferative zone cell size in the 4-5- month-old miniature pigs that we used in our model (Noonan et al. 1998) is smaller. Hence, 5.0 times cell height increase in the model is from 5.6 to $28.1 \mu \mathrm{m}$, with 


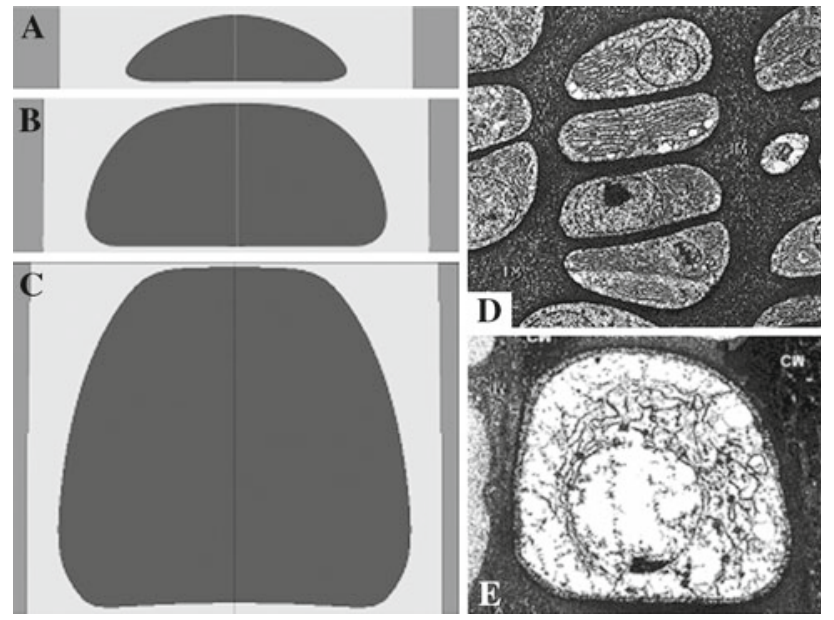

Fig. 4 Cell shapes in simulations and measurements (Hunziker 1994). The initial cell shape used in the model, derived from 4- to 5-monthold miniature pig tibia (Noonan et al. 1998), is relatively flat (a). The geometry of early (b) and late hypertrophic (c) cells concur qualitatively with the appearance of late proliferative (d) and terminally differentiated cells (e) in 35-day-old rat tibia ( $\mathbf{d}$ and $\mathbf{e}$ adapted from Hunziker et al. (1994))

cell volume being 15 times that of the proliferative zone cell. For qualitative comparison of cell shapes between simulation and experiment (Hunziker 1994), hypertrophy was extended such that the cell reached $38 \mu \mathrm{m}$ (Fig. 4). Both in the simulation and the experimental data, ECM volume increased 2.7-fold from proliferative to hypertrophic. The simulation predicts that PCM increases 3.0-fold, while ITM increases to only 1.9 times its original volume. Unfortunately, this difference between ITM and PCM cannot be verified with experimental data. Another study documented that a chondrocyte with tenfold increased volume will have gained four times its original cell height (Hunziker and Schenk 1989), which is in agreement with the prediction that a cell that volume reaches 3.6-fold the original cell height.

Modulation of bone growth by external compression or tension (Hueter-Volkmann principle) is known to affect hypertrophy. In simulations, additional tensions of $0.1,0.05$, -0.05 and $-0.1 \mathrm{MPa}$ are applied in the direction of growth, to enable comparison with experimental studies (Robling et al. 2001; Stokes et al. 2006, 2007). Ultimate chondrocyte height, representative for bone growth rate (Wilsman et al. 1996), correlates with applied tension. Compression (8.2\% reduction of cell height at $-0.1 \mathrm{MPa}$ loading) is predicted to be slightly less effective than tension $(12.2 \%$ enhanced growth at $+0.1 \mathrm{MPa}$ loading) in modulating hypertrophy with the default parameter set. This concurs with averaged changes in hypertrophic cell size between rat, calve and rabbit versus sham-operated animals, which show lowered final cell size during $0.1 \mathrm{MPa}$ compression by $5 \%$ (tibia) or $7 \%$ (vertebra) and enlarged sizes during $0.1 \mathrm{MPa}$ tension by $15 \%$ (tibia) or $8 \%$ (vertebra) (Stokes 2002; Stokes et al. 2005). In another study, continuous $0.1 \mathrm{MPa}$ compression reduced final chondrocyte size in rat tibiae $7 \%$ and in vertebrae $12 \%$ (Stokes 2002; Stokes et al. 2005). Upon increasing the turnover rate of collagen, hypertrophy is faster and ultimate chondrocyte height increases. The latter two are known to correlate (Breur et al. 1991; Hunziker and Schenk 1989; Hunziker et al. 1987; Kuhn et al. 1996), but this has so far not been studied in relation with collagen turnover rate. Another interesting but unverifiable model prediction is that at higher collagen turnover rates, hypertrophy becomes more susceptible to external loading.

When proteoglycan expression is fully compromised or reduced to half the normal amount, ultimate cell height decreases by $3 \%$ (partially compromised) or $7 \%$ (fully compromised), while nearest axial distance between two hypertrophic cells decreases by $22 \%$ (partially compromised) or $41 \%$ (fully compromised) and cell-to-cell-center distance changed 5\% (partially compromised) or 9\% (fully compromised). Although experimentally observed cell height reductions under these conditions exceed our predictions, Gakunga et al. (2000) and Vanky et al. (2000) also show reduced hypertrophy when hyaluronan is absent $(\sim 20 \%)$, reduced $(\sim 18 \%)$, or not well retained in the tissue $(\sim 10 \%)$.

Finally, when collagen turnover by MMP's is prohibited, simulated by omitting Eq. 25, yet leaving all other parameters unchanged, cells slightly increase volume but do not hypertrophy. The intercellular space narrows, even though ECM continues to be expressed, a picture that concurs with the histological appearance of the hypertrophic zone in S1Pcho mice, which cannot enzymatically degrade collagen-II (Patra et al. 2006a,b) (Fig. 5). Unfortunately, thorough quantitative comparison is not possible for the lack of experimental data. However, the model predicts that cell and tissue strive to grow, but growth is prohibited by stress in the collagen fibers, which raises to eightfold the stress in the simulation with normal collagen remodeling. Concurrently, intracellular pressure doubles, and extracellular pressure rises threefold.

\section{Discussion}

This study contains three parts. The first part shows that reported changes in tissue constitution, when translated into mechanical effects, induce cell hypertrophy. Noteworthy, cell volume, fibrillar and non-fibrillar matrix volumes in both pericellular and extracellular area, match exactly between experimental observations and simulations. The only unknown parameter that was fitted to obtain this match was intracellular charged protein content. With that, we show for the first time that it is possible to capture hypertrophy in a fully sound mechanical evaluation. In the second part, we gain further confidence in this mechanical approach. We show that extended hypertrophy results in a cell shape and matrix 
Fig. 5 Midsaggittal section through the proximal tibia of an E14.5 wild-type (a) and S1Pcko (b) mouse, with inserts in the early proliferative and hypertrophic zones representing the chondrocyte shape as predicted in simulations (Simulations: red ITM, green PCM, blue chondrocyte. Histology: Saffranin-O/Fast Green/Hematoxilin staining. The image was kindly provided by dr D. Patra and prof L.J. Sandell

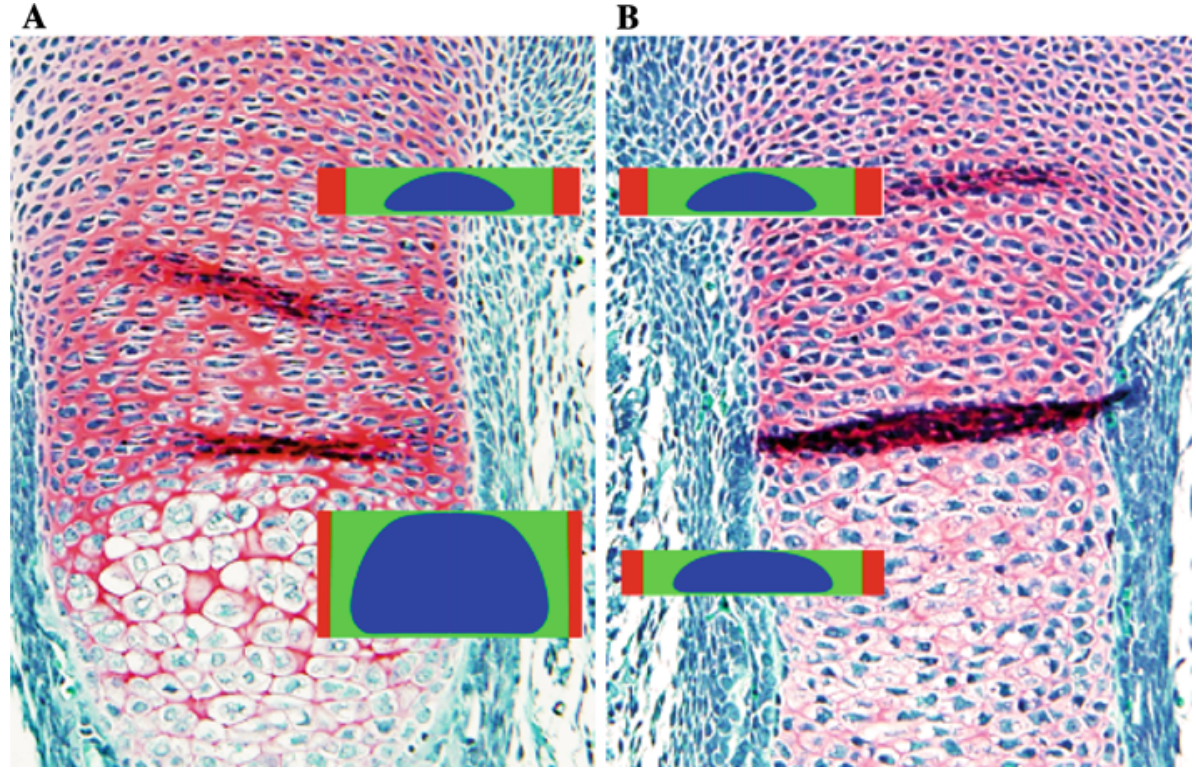

characteristics similar to those described in other species. Also, we show that the Hueter-Volkmann principle can be a direct result of mechanical conditions, rather than an effect that requires differential active cell responses. In addition, concentration-dependent effects of partially or fully compromised hyaluronan expression by cells corroborate well, qualitatively. In the last part of the study, we evaluate the S1Pcko knockout mouse model from a mechanical perspective. This mouse cannot degrade collagen-II, contains chondrocytes that appear pressurized, but are unable to hypertrophy even though they express all markers known to induce hypertrophy. We explain this by showing that if collagen turnover is compromised, the tension that develops in the collagen network increases dramatically and is sufficient to withstand cell growth.

One asset of this study is that all simulations are performed with the same mechanical parameters, which are mostly derived from one descriptive study (Noonan et al. 1998). These parameters are subsequently applied to evaluate various pathophysiological conditions. Simulations corroborate experimental data surprisingly well, even though these involve different species, ages and joints. To appropriately compare quantitative effects between experiments sometimes requires normalizing the effects. We prefer this approach over adjusting numerical parameters per case, because it shows the general applicability of the mechanical principles that are considered.

Cell swelling is modeled using a Donnan equilibrium analysis, instead of adopting a mechanism of cell swelling due to partitioning of membrane-impermeant osmolytes. Regardless of the mechanism behind accumulation of ion concentration, the net effect is cell swelling. We fit the two unknown cellular parameters $\mathrm{d} V_{\mathrm{PG}}$ and $c_{\mathrm{PG}}$, for cell swelling (Table 1).
This results in apparent intracellular fixed charge densities between 0.1 and $0.2 \mathrm{meq} / \mathrm{ml}$, depending on the level of cell maturation. These values are within the previously reported range (Julkunen et al. 2009; Lai et al. 2002; Likhitpanichkul et al. 2005).

In simulations with compromised collagen turnover, intracellular fixed charge density increased to $0.6 \mathrm{meq} / \mathrm{ml}$. Whether or not this occurs inside the cells of S1Pcko mice is unknown and challenging to determine. However, if such high pressures would arise, they would likely be detrimental to the cell. Indeed, S1Pcko mice show severe intracellular changes, exemplified by severely disrupted endoplasmic reticulum and a pathological fat-metabolism (Patra et al. 2007). However, causality remains speculative.

The implementation of collagen turnover requires further evaluation. First, several collagen fiber departments were defined and these were assumed to turnover sequentially. Hence, it was assumed that older fibers would be more susceptible to damage than younger ones. Although this is a simplification of a complex mechanism, the assumption may hold to some extent. The likelihood of enzymatic collagen degradation is minimal at 4-5\% fibril strain (Huang and Yannas 1977), or at around $3 \mathrm{pN}$ per collagen monomer (Ruberti and Hallab 2005). During hypertrophy, the tissue elongates and the strain in the collagen fibers increases beyond the protective strain level, making older fibers more susceptible to degradation. Second, turnover is approached as a mechanism where old collagen fibers are degraded and replaced by new, unstrained ones. An alternative explanation is that fibrils partly turn over and adjust to the level of strain they experience by increasing the number of serial monomers, theoretically resulting in a longer fibril under unloaded conditions. Such mechanism is likely 
to keep stresses in growing soft tissues low (Foolen et al. 2009). The present simulations are indifferent to the exact mechanism of collagen adaptation. The only premise is that fibers experiencing excessive mechanical strain are replaced by unstrained fibers. Third, collagen fibers are assumed to assemble in the direction of tissue elongation in the ITM, while there is no clear anisotropic organization in the PCM. This is a feasible assumption, given the orientation of fibers visible in EM images of growth plates (Hunziker 1994; Noonan et al. 1998) and the alignment with growth observed in other embryonic soft tissue (Foolen et al. 2008). Also, collagen orientation in cartilage is known to align with the direction of principal strain (Wilson et al. 2006a), which equals the direction of growth.

In conclusion, it is shown that hypertrophy can be the result of a mechanical interplay between intracellular pressure induced by the cell, tissue pressures induced by proteoglycans, and collagen turnover. The latter turns out to be critical for hypertrophy. Although differential active metabolic responses by chondrocytes in response to perturbations cannot be excluded, this paper shows that the mechanical conditions alone can explain a variety of experimentally observed effects. Availability of quantitative data has been critical to develop and validate our model and to reach these conclusions. Therefore, most data have been derived from hypertrophy in the epiphyseal plate, which has been extensively studied. However, the mechanical principles that we exposed are general. We therefore expect the present work to be applicable to hypertrophying chondrocytes in osteoarthritic cartilage as well.

Open Access This article is distributed under the terms of the Creative Commons Attribution Noncommercial License which permits any noncommercial use, distribution, and reproduction in any medium, provided the original author(s) and source are credited.

\section{References}

Alexopoulos LG, Haider MA, Vail TP, Guilak F (2003) Alterations in the mechanical properties of the human chondrocyte pericellular matrix with osteoarthritis. J Biomech Eng 125:323-333

Alini M, Matsui Y, Dodge GR, Poole AR (1992) The extracellular matrix of cartilage in the growth plate before and during calcification: changes in composition and degradation of type II collagen. Calcif Tissue Int 50:327-335

Breur GJ, Vanenkevort BA, Farnum CE, Wilsman NJ (1991) Linear relationship between the volume of hypertrophic chondrocytes and the rate of longitudinal bone growth in growth plates. J Orthop Res 9:348-359

Buckwalter JA, Mower D, Ungar R (1986) Morphometric analysis of chondrocyte hypertrophy. J Bone Joint Surg Ser A 68:243-255

Farnum CE, Lee R, O'Hara K, Urban JPG (2002) Volume increase in growth plate chondrocytes during hypertrophy: the contribution of organic osmolytes. Bone 30:574-581

Foolen J, van Donkelaar C, Nowlan N, Murphy P, Huiskes R, Ito $\mathrm{K}$ (2008) Collagen orientation in periosteum and perichondrium is aligned with preferential directions of tissue growth. J Orthop Res 26:1263-1268

Foolen J, van Donkelaar CC, Murphy P, Huiskes R, Ito K (2009) Residual periosteum tension is insufficient to directly modulate bone growth. J Biomech 42:152-157

Gakunga PT, Kuboki Y, Opperman LA (2000) Hyaluronan is essential for the expansion of the cranial base growth plates. J Craniofacial Genet Dev Biol 20:53-63

Guilak F, Alexopoulos L, Haider M, Ting-Beall H, Setton L (2005) Zonal uniformity in mechanical properties of the chondrocyte pericellular matrix: micropipette aspiration of canine chondrons isolated by cartilage homogenization. Ann Biomed Eng 33:13121318

Guilak F, Erickson GR, Ting-Beall HP (2002) The effects of osmotic stress on the viscoelastic and physical properties of articular chondrocytes. Biophys J 82:720-727

Huang C, Yannas IV (1977) Mechanochemical studies of enzymatic degradation of insoluble collagen fibers. J Biomed Mater Res Part A 11:137-154

Hunziker EB (1994) Mechanism of longitudinal bone growth and its regulation by growth plate chondrocytes. Microsc Res Tech 28:505-519

Hunziker EB, Schenk RK (1989) Physiological mechanisms adopted by chondrocytes in regulating longitudinal bone growth in rats. J Physiol 414:55-71

Hunziker EB, Schenk RK, Cruz-Orive LM (1987) Quantitation of chondrocyte performance in growth-plate cartilage during longitudinal bone growth. J Bone Joint Surg Am Vol 69:162-173

Huyghe JM, Houben GB, Drost MR, van Donkelaar CC (2003) An ionised/non-ionised dual porosity model of intervertebral disc tissue. Biomech Model Mechanobiol 2:3-19

Julkunen P, Wilson W, Jurvelin J, Korhonen R (2009) Composition of the pericellular matrix modulates the deformation behaviour of chondrocytes in articular cartilage under static loading. Med Biol Eng Comput 47:1281-1290

Kirsch T, Swoboda B, Nah H (2000) Activation of annexin II and $\mathrm{V}$ expression, terminal differentiation, mineralization and apoptosis in human osteoarthritic cartilage. Osteoarthr Cartil 8:294302

Kuhn JL, DeLacey JH, Leenellett EE (1996) Relationship between bone growth rate and hypertrophic chondrocyte volume in New Zealand white rabbits of varying ages. J Orthop Res 14:706711

Kuizon B, Salusky I (2004) Growth plate disturbances in renal failure. Clin Rev Bone Miner Metab 2:53-61

Kumagishi K, Nishida K, Momota R, Yamaai Y, Hirohata S, Demircan K, Naito I, Ninomiya Y, Ohtsuka A (2008) Expression of ADAMTS-9 in mouse growth plate cartilage. Matrix Biol 27:24-25

Lai WM, Sun DD, Ateshian GA, Guo XE, Mow VC (2002) Electrical signals for chondrocytes in cartilage. Biorheology 39:39-45

Likhitpanichkul M, Guo XE, Mow VC (2005) The effect of matrix tension-compression nonlinearity and fixed negative charges on chondrocyte responses in cartilage. Mol Cell Biomech 2:191-204

Matsui Y, Alini M, Webber C, Poole AR (1991) Characterization of aggregating proteoglycans from the proliferative, maturing, hypertrophic, and calcifying zones of the cartilaginous physis. J Bone Joint Surg Am Vol 73:1064-1074

Maroudas A, Bannon C (1981) Measurement of swelling pressure in cartilage and comparison with the osmotic pressure of constituent proteoglycans. Biorheology 18:619-632

Maroudas A, Wachtel E, Grushko G, Katz EP, Weinberg P (1991) The effect of osmotic and mechanical pressures on water partitioning in articular cartilage. Biochem Biophys Acta 1073:285-294

Narmoneva DA, Wang JY, Setton LA (1999) Nonuniform swellinginduced residual strains in articular cartilage. J Biomech 32:401408 
Noonan KJ, Hunziker EB, Nessler J, Buckwalter JA (1998) Changes in cell, matrix compartment, and fibrillar collagen volumes between growth-plate zones. J Orthop Res 16:500-508

Ortega N, Behonick DJ, Werb Z (2004) Matrix remodeling during endochondral ossification. Trends Cell Biol 14:86-93

Patra D., Xing X, Bryan J, Sandell LJ (2006a) Abnormal matrix in S1Pcko mice leads to lack of bone formation. Matrix Biol 25:S71

Patra D, Davies , Bryan J., Xing X, Sandell LJ (2006b) Lack of endochondral ossification in site-1 protease knockout mice. Eur Cells Mater 12:8

Patra D, Xing X, Davies S, Bryan J, Franz C, Hunziker EB, Sandell LJ (2007) Site-1 protease is essential for endochondral bone formation in mice. J Cell Biol 179:687-700

Radhakrishnan P, Lewis NT, Mao JJ (2004) Zone-specific micromechanical properties of the extracellular matrices of growth plate cartilage. Ann Biomed Eng 32:284-291

Robling AG, Duijvelaar KM, Geevers JV, Ohashi N, Turner CH (2001) Modulation of appositional and longitudinal bone growth in the rat ulna by applied static and dynamic force. Bone 29:105113

Ruberti JW, Hallab NJ (2005) Strain-controlled enzymatic cleavage of collagen in loaded matrix. Biochem Biophys Res Commun 336:483-489

Saarakkala S, Julkunen P, Kiviranta P, M $\Sigma$ kitalo J, Jurvelin JS, Korhonen RK (2010) Depth-wise progression of osteoarthritis in human articular cartilage: investigation of composition, structure and biomechanics. Osteoarthr Cartil 18:73-81

Stokes IAF (2002) Mechanical effects on skeletal growth. J Musculoskelet Neuronal Interact 2:277-280

Stokes IAF, Aronsson DD, Dimock AN, Cortright V, Beck S (2006) Endochondral growth in growth plates of three species at two anatomical locations modulated by mechanical compression and tension. J Orthop Res 24:1327-1334

Stokes IAF, Clark KC, Farnum CE, Aronsson DD (2007) Alterations in the growth plate associated with growth modulation by sustained compression or distraction. Bone 41:197-205

Stokes IA, Gwadera J, Dimock A, Farnum CE, Aronsson DD (2005) Modulation of vertebral and tibial growth by compression loading: diurnal versus full-time loading. J Orthop Res 23:188-195

Sztrolovics R, Recklies AD, Roughley PJ, Mort JS (2002) Hyaluronate degradation as an alternative mechanism for proteoglycan release from cartilage during interleukin-1beta-stimulated catabolism. Biochem J 362:473-479

Tchetina EV, Kobayashi M, Yasuda T, Meijers T, Pidoux I, Poole AR (2007) Chondrocyte hypertrophy can be induced by a cryptic sequence of type II collagen and is accompanied by the induction of MMP-13 and collagenase activity: implications for development and arthritis. Matrix Biol 26:247-258

Urban JPG, McMullin JF (1988) Swelling pressures of the lumbar intervertebral discs: influence of age, spinal level, composition and degeneration. Spine 13:179-187

van der Kraan PM, Blaney Davidson EN, Blom A, van den Berg WB (2009) TGF-beta signaling in chondrocyte terminal differentiation and osteoarthritis: modulation and integration of signaling pathways through receptor-Smads. Osteoarthr Cartil 17:1539-1545

Vanky P, Brockstedt U, Nurminen M, Wikstrom B, Hjerpe A (2000) Growth parameters in the epiphyseal cartilage of brachymorphic $(\mathrm{bm} / \mathrm{bm})$ mice. Calcif Tissue Int 66:355-362

Wilkie AOM (2005) Bad bones, absent smell, selfish testes: the pleiotropic consequences of human FGF receptor mutations. Cytokine Growth Factor Rev 16:187-203

Williamson AK, Chen AC, Sah RL (2001) Compressive properties and function-composition relationships of developing bovine articular cartilage. J Orthop Res 19:1113-1121

Wilsman NJ, Farnum CE, Leiferman EM, Fry M, Barreto C (1996) Differential growth by growth plates as a function of multiple parameters of chondrocytic kinetics. J Orthop Res 14:927-936

Wilson W, Driessen NJB, Van Donkelaar CC, Ito K (2006) Prediction of collagen orientation in articular cartilage by a collagen remodeling algorithm. Osteoarthr Cartil 14:1196-1202

Wilson W, Huyghe JM, Van Donkelaar CC (2006) A compositionbased cartilage model for the assessment of compositional changes during cartilage damage and adaptation. Osteoarthr Cartil 14:554 560

Wilson W, Huyghe JM, Van Donkelaar CC (2007) Depth-dependent compressive equilibrium properties of articular cartilage explained by its composition. Biomech Model Mechanobiol 6:43-53

Wilson W, Van Donkelaar CC, Van Rietbergen B, Huiskes R (2005) A fibril-reinforced poroviscoelastic swelling model for articular cartilage. J Biomech 38:1195-1204

Wilson W, Van Donkelaar CC, Van Rietbergen B, Ito K, Huiskes $\mathrm{R}$ (2004) Stresses in the local collagen network of articular cartilage: a poroviscoelastic fibril-reinforced finite element study. J Biomech 37:357-366

Zuscik MJ, Pateder DB, Puzas JE, Schwarz EM, Rosier RN, O'Keefe RJ (2002) Lead alters parathyroid hormone-related peptide and transforming growth factor-beta1 effects and AP-1 and NF-kappaB signaling in chondrocytes. J Orthop Res 20:811-818 